\title{
Recurrent stroke: the role of thrombophilia in a large international pediatric stroke population
}

\begin{abstract}
Gabrielle deVeber, ${ }^{1 *}$ Fenella Kirkham, ${ }^{2,3 *}$ Kelsey Shannon, ${ }^{1}$ Leonardo Brandão, ${ }^{1}$ Ronald Sträter, ${ }^{4}$ Gili Kenet,${ }^{5}$ Hartmut Clausnizer, ${ }^{6}$ Mahendranath Moharir, ${ }^{1}$ Martina Kausch, ${ }^{6}$ Rand Askalan, ${ }^{1}$ Daune MacGregor, ${ }^{1}$ Monika Stoll, ${ }^{7}$ Antje Torge, ${ }^{6}$ Nomazulu Dlamini, ${ }^{1}$ Vijeja Ganesan, ${ }^{2}$ Mara Prengler, ${ }^{2}$ Jaspal Singh ${ }^{3}$ and Ulrike Nowak-Göttl ${ }^{4 / 6}$
\end{abstract}

${ }^{1}$ The Hospital for Sick Children, Toronto, Canada; ${ }^{2}$ Developmental Neurosciences Programme, UCL Great Ormond Street Institute of Child Health, London, UK; ${ }^{3}$ University Hospital Southampton, UK; ${ }^{4}$ Department of Paediatric Haematology/Oncology, University of Münster, Münster, Germany; ${ }^{5}$ Pediatric Coagulation Service, National Hemophilia Centre and Institute of Thrombosis and Hemostasis Sheba Medical Center, Tel-Hashomer, Israel; ${ }^{I}$ Institute of Clinical Chemistry, University Hospital Kiel-Lübeck, Kiel, Germany and ${ }^{7}$ Department of Genetic Epidemiology, University of Münster, Münster, Germany

*GdeV and FK contributed equally to this work.

In the article pre-published online on January 24, 2019 and published in the paper version of Haematologica [volume 104(8):1676-1681; doi:10.3324/haematol2018.211433] we have to correct:

$>$ that recurrent stroke occurred in 160/872 (instead of $160 / 880$ ) children [page 1678, second column, line 6].

$>$ the incidence rates of recurrent AIS with respect to the individual exposure time in years given in the abstract (page 1676, lines 16-18) and in the results section (page 1679, paragraph "prothrombotic risk factors", lines 38-40). As explained in the methods section, we calculated the absolute risk of AIS recurrence as incidence rates per 100 patient-years (\%). According to the individual exposure times (years) to antithrombin, lipoprotein (a) and the presence of more than one prothrombotic risk factor the incidence rates calculated per $\mathbf{1 0 0}$ patient-years are presented in the table below.

\begin{tabular}{lccc}
\hline IJpe of & $\begin{array}{c}\text { Exposure time } \\
\text { (Jears) }\end{array}$ & $\begin{array}{c}\text { Event (n) } \\
\text { thrombophilia }\end{array}$ & $\begin{array}{c}\text { Incidence per 100 patient-years } \\
\%(95 \% \text { Cl) }\end{array}$ \\
Antithrombin deficiency & 2437 & 7 & $0.3(0.1-0.6)$ \\
Elevated lipoprotein(a) & 1938 & 23 & $1.2(0.7-1.7)$ \\
\hline More than one prothrombotic risk factor & 2887 & 23 & $0.8(0.5-1.1)$ \\
\hline
\end{tabular}

CI: Confidence interval; n: number. 